\title{
Paraneoplastic dermatomyositis presenting myopathy combined with synchronous cervical and sigmoid colon cancer
}

Jae Heun Chung ${ }^{1}$, Min Wook $\mathrm{So}^{1}$, and Seong-Geun Kim ${ }^{1,2}$

\author{
${ }^{1}$ Department of Internal Medicine, \\ ${ }^{2}$ Research Institute for Convergence \\ of Biomedical Science and \\ Technology, Pusan National \\ University Yangsan Hospital, \\ Yangsan, Korea
}

Received: October 31, 2014

Revised : February 24, 2015

Accepted: March 3, 2015

\section{Correspondence to}

Seong-Geun Kim, M.D.

Division of Hemato-Oncology,

Department of Internal Medi-

cine, Pusan National University

Yangsan Hospital, 20 Geumo-ro,

Mulgeum-eup, Yangsan 50612,

Korea

Tel: $+82-55-360-1425$

Fax: +82-55-360-1425

E-mail: sgkimpatheny@gmail.com
To the Editor,

Dermatomyositis (DM) is an idiopathic inflammatory connective-tissue disease characterized by inflammation of the skin and muscles. The hallmark of this disorder is muscle weakness. In addition, the disorder is associated with a variety of laboratory findings and clinical manifestations: clinical inflammatory dermatological manifestations (Gottron's sign and heliotrope rash), electromyogram (EMG) abnormalities, histopathologic muscle abnormalities, serum muscle enzyme elevation, and serum autoantibodies. DM is often associated with a high incidence of malignancy, such as adenocarcinoma of the lung, ovary, pancreas, bladder, and stomach. Treatment of DM in a patient with cancer constitutes the management of cancer and DM. Immunosuppressive therapy (high-dose corticosteroids and nonsteroidal drugs such as methotrexate, azathioprine, and cyclosporine) may control the skin lesions occurring in the presence of symptomatic muscle weakness. Intravenous immunoglobulin (IVIG) is a second-line therapy for patients with steroid-resistant disorders.

A 49-year-old woman who presented with a high-grade squamous intraepithelial lesion and positive human papillomavirus (HPV; genotype 31, 55) found on a routine pap smear performed at the local gynecology clinic was referred to our hospital. She was a nonsmoker and was not addicted to alcohol. She had no significant past gynecological history, but her father was positive for gastric cancer. The patient had never presented any symptoms or laboratory abnormalities due to rheumatologic or neurologic disease.

The diagnosis was determined by punch biopsy of the cervix that revealed cervical intraepithelial neoplasia grade III. This was followed by a loop electrical excision procedure (LEEP) on the cervix. The cervical tissue obtained from the LEEP showed squamous cell carcinoma in situ. Four weeks after diagnosis, she was admitted for total laparoscopic hysterectomy. The tissue biopsy obtained from hysterectomy showed no residual tumor after the cervical conization.

One month after discharge, the patient visited the emergency room with myalgia, weakness of the shoulder and pelvic girdle muscle, and cutaneous disease. On examination, she had erythema on the upper eyelid, neck and trunk, facial edema, and Gottron's papules on the fingers (Fig. 1). Serum examination showed high levels of creatine kinase (11,744 U/L) and aldolase (67.7 $\mathrm{IU} / \mathrm{L})$. Immunological investigations 


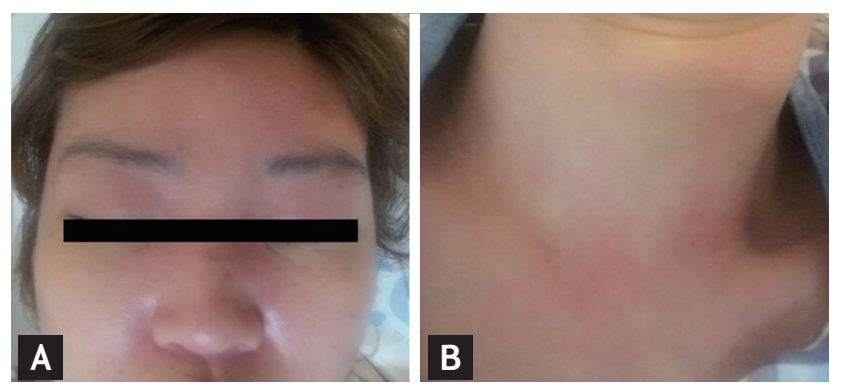

Figure 1. Clinical pictures. (A) Edema of the eyelids and diffuse erythematous papules were seen on the forehead, and nasal bridge. (B) Erythema of the neck.

showed positive antinuclear antibody $(1: 2,560)$ with negative anti-JO-1, anti-dsDNA, anti-Smith antibodies, anti-Ro, and anti-La, while complement levels were normal. Magnetic resonance imaging (MRI) of the pelvis showed multifocal high signal areas with enhancement at the gluteus, obturator, quadrates femoris, vastus, and gracilis muscles (Fig. 2A). Electromyography showed increased insertional activities, fibrillation potentials, and positive sharp waves in the biceps, deltoid, extensor digitorum communis, gastrocnemius medius, tibialis anterior, and vastus lateralis muscles indicating active myopathy. Skin biopsy from an erythematous lesion on the dorsal hand showed perivascular lymphocytic infiltration. Muscle biopsy from the biceps muscle revealed a perifascicular atrophy pattern (Fig. 2B). Based on the clinical characteristics and laboratory findings, we diagnosed the patient with cervical cancer-associated DM.

Treatment was initiated with intravenous methylprednisolone ( $60 \mathrm{mg} /$ day). The result was partial remission of skin rash, but extremity weakness on exertion and skeletal muscle pain did not respond to the treatment.

During admission, the patient demonstrated progressive extremity weakness and myalgia. The serum creatine kinase level remained elevated (13,001 U/L). Abnormal liver function test results were obtained: elevated aspartate aminotransferase (662 IU/L) and elevated alanine aminotransferase (325 IU/L). A computed tomography (CT) scan of the abdomen showed a round hypodense liver mass (Fig. 3A), but chest CT showed no evidence of thoracic metastasis. Serum tumor markers carcinoembryonic antigen and carbohydrate antigen 19-9 increased to $18.11 \mathrm{ng} / \mathrm{mL}$ (reference range, o to 5.0) and 190.17 U/ $\mathrm{mL}$ (reference range, o to 39), respectively. Liver biopsy was performed and indicated the presence of metastatic

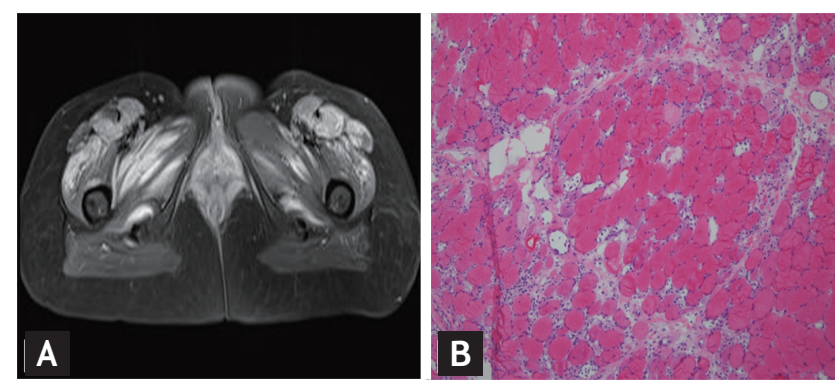

Figure 2. Magnetic resonance imaging and pathological findings. (A) T2-weighted images through the proximal thigh. Increased signal intensity was seen in the gluteus, obturator, quadrates femoris, vastus, and gracilis muscles. (B) Microscopic view $(H \& E, \times 100)$ revealed perifascicular atrophy pattern of biceps muscle.

adenocarcinoma, probably originating from the colon. Positron emission tomography (PET) scans showed sigmoid colon cancer with pericolic lymph node metastasis and a lesion in the right lobe of the liver (Fig. $3 \mathrm{~B}$ and $\left.{ }_{3} \mathrm{C}\right)$. Biopsy taken at colonoscopy revealed the presence of adenocarcinoma of the sigmoid colon. The patient underwent right hepatectomy, cholecystectomy, and laparoscopic anterior resection. Subsequent adjuvant chemotherapy with leucovorin, 5-fluorouracil, and oxaliplatin (FOLFOX6 regimen) was performed for 8 cycles. After recovering from surgery, the patient was discharged with oral steroid agents to be administered at our out-patient rheumatic clinic. The steroid treatment was slowly tapered over 6 months by our rheumatologist.

Four months after adjuvant chemotherapy, the patient was readmitted to the hospital because of progressive facial edema, general myalgia, and lower extremity weakness, especially, when walking. Several laboratory results supported the manifestations of DM with increases in creatine kinase $(20,268 \mathrm{U} / \mathrm{L})$, serum myoglobin $(2,037 \mathrm{ng} /$ $\mathrm{mL})$, serum lactate dehydrogenase (3,170 IU/L), and erythrocyte sedimentation rate (ESR; $23 \mathrm{~mm} / \mathrm{hr}$ ). Although the patient was treated with IV methylprednisolone $(60 \mathrm{mg} /$ day) over 2 weeks, she showed no improvement in serum creatine kinase, myoglobin, or clinical manifestations.

Therefore, she was started on IVIG $(500 \mathrm{mg} / \mathrm{kg}$ for 4 days every 4 weeks for 3 months) and steroid at a tapered dose. Extremity weakness and muscular symptoms regressed after 3 months of treatment. The cutaneous lesions, including Gottron's papules, disappeared within 8 weeks. Serum creatine kinase, ESR, and lactate dehydrogenase decreased to $237 \mathrm{U} / \mathrm{L}, 13 \mathrm{~mm} / \mathrm{hr}$, and $569 \mathrm{IU} / \mathrm{L}$, 

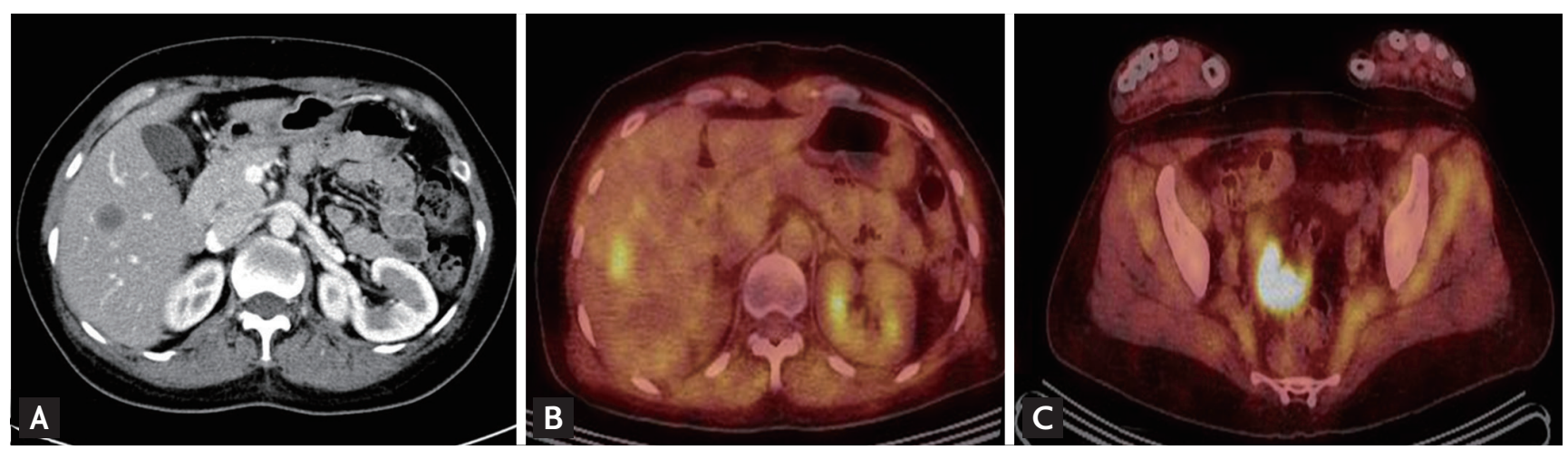

Figure 3. Computed tomography/positron emission tomography scans. (A) Round hypodense mass, $1.8 \mathrm{~cm}$, at the liver $\mathrm{S}_{5}$. (B) Hypermetabolic lesion in liver right lobe and $(\mathrm{C})$ sigmoid colon cancer with pericolic lymph nodes metastasis.

respectively, after 8 weeks of treatment. PET/CT scans showed no evidence of local recurrence or distant metastasis. The patient had a relapse-free survival until 15 months after the initial diagnosis.

The initial diagnosis in our patient was aimed at muscle involvement, such as proximal weakness, and increase in creatine kinase levels. In addition, the T2-weighted MRI showed increased signal intensity of muscles in the lower extremities. Concurrently, muscle biopsy showed the perifascicular atrophy pattern. Neither the patient nor her family had a previous history of muscle disease. Lambert-Eaton syndrome, a relatively common neuromuscular disease, was excluded, because its typical symptoms, such as worsening after exercise or autonomic dysfunction, were not found in our patient. In addition, EMG showed no signs of peripheral neuropathy or injury of the neuromuscular junction or the muscle. Therefore, the diagnosis of DM was established.

To date, DM has reportedly been associated with various types of cancers. High prevalence of cancer in the ovary, lung, and gastrointestinal tract has been reported from Western countries [1]. Malignancy can be diagnosed before, concurrent with, or after the diagnosis of DM. In our case, the symptoms of DM newly erupted after surgical treatment of the patient with sigmoid colon cancer. DM symptoms can occur after the diagnosis of cancer, and we initially postulated the hypothesis that cervical cancer might induce the paraneoplastic DM. However, the improvement of muscle weakness was not shown in spite of the treatment of underlying neoplasm. Thus, the relationship between squamous cell carcinoma of cervix and paraneoplastic DM was not clear. Further work-up showed that the muscular symptoms worsened before the discovery of sigmoid colon cancer with liver metastasis. Therefore, we judged that this patient's DM may be elicited by sigmoid colon cancer. Limited studies of sigmoid colon cancer have been reported to date. Moreover, only two cases in the Korean literature on colon cancer-related DM have been reported, to the best of our knowledge. The DM in 1 case was associated with cancer of an unknown primary site, possibly the colon, and DM involuted after treatment with high dose steroid and methotrexate [2]. The other case presented with acute renal failure associated DM and colon cancer [3]. However, this article did not show a relationship between cancer and DM. On the contrary, our patient showed sigmoid cancer-associated DM. In addition, our patient underwent steroid-resistant treatment with IVIG, which could be expected to resolve muscular symptoms and cutaneous manifestation, and thus, markedly downregulate creatine kinase.

To date, corticosteroids remain the initial agent of choice in the treatment of DM. However, in steroid-resistant cases like ours, treatment can be combined with or replaced by second-line agents such as IVIG. We are aware of limited studies conducted in patients with paraneoplastic DM that have demonstrated IVIG to be ineffective in cases similar to ours, even in combination with corticosteroids [4]. In comparison with those observations, our patient responded to IVIG treatment, which could be considered an active agent involved in treatment of patients who are resistant to steroid therapy. In addition, the small number of existing studies revealed that the symptoms and clinical findings of DM were im- 
proved following surgical therapy or with adjuvant chemotherapy for cancer treatment. Cox et al. [5], for example, reported patients who showed improvement in their DM with tumor resection and DM relapses concurrent with recurrent tumor. The improvement of our patient's DM was not followed by surgical therapy for cervical carcinoma or sigmoid colon cancer with hepatectomy.

Altogether, this case report is a unique description of a myopathy involved in the course of sigmoid colon cancer-related DM, associating successful treatment with IVIG against the refractory aspects of conventional therapy.

Keywords: Dermatomyositis; Muscular disease; Neoplasms

\section{Conflict of interest}

No potential conflict of interest relevant to this article was reported.

\section{REFERENCES}

1. Hill CL, Zhang Y, Sigurgeirsson B, et al. Frequency of specific cancer types in dermatomyositis and polymyositis: a population-based study. Lancet 2001;357:96-100.

2. Kim HI, Chung SH, Hwang JE, et al. Dermatomyositis associated with cancer of unknown primary site. J Korean Med Sci 2007;22 Suppl:S174-S177.

3. Park SK, Cho KH, Kang SK. Acute renal failure associated with dermatomyositis and colon cancer. Nephron 1990;55:225-226.

4. Sadayama T, Miyagawa S, Shirai T. Low-dose intravenous immunoglobulin therapy for intractable dermatomyositis skin lesions. J Dermatol 1999;26:457-459.

5. Cox NH, Lawrence CM, Langtry JA, Ive FA. Dermatomyositis: disease associations and an evaluation of screening investigations for malignancy. Arch Dermatol 1990;126:61-65. 